\title{
DERECHO, MORAL Y EL PROBLEMA DE LA NO IDENTIDAD: APUNTES SOBRE EL CONCEPTO DE DAÑO *
}

\author{
Santiago Truccone Borgogno **
}

CONICET

RESUMEN. En el presente escrito intentaré explorar la relación entre dos temas controvertidos: el daño y el problema de la no-identidad. Sostendré la idea de que ninguna tesis plausible del daño puede resolver completamente el problema de la no-identidad. Sin embargo, defenderé que una reformulación de la tesis comparativa contra-fáctica es superior a todas las otras tesis del daño y por tanto debería ser adoptada.

Palabras clave: Daño, problema de la no-identidad, comparativismo, PARFIT.

\section{Law, Moral and the Non-Identity Problem: Notes about the Concept of Harm}

ABSTRACT. In this paper I tried to explore the relation between two controversial issues: harm and the non-identity problem. I will defend the idea that any harm account cannot fully solve the nonidentity problem. However, I will defend that a reformulation of the counterfactual comparative account is better than all others harm accounts, and therefore should be adopted.

Keywords: Harm, non-identity problem, comparativism, PARFIT.

* Fecha de recepción: 29 de abril de 2015. Fecha de aceptación: 23 de julio de 2015.

** Agradezco a Romina Frontalini ReKERS y Ramiro MOYAnO con quienes he discutido reiteradas veces el argumento de este trabajo. Debo agradecer especialmente a Hugo SELEME, José PerAlta, Miguel A. RoDILLA y Guillermo LARIGUET, por sus valiosas objeciones. También a Fabián BALCARCE, Rodrigo SÁNCHEZ BRÍGIDO, Cristina Méndez Rodríguez, Esteban Llamosas, Tim Meijers, Juan Iosa, Matías Gonzales del Solar y a Jorge Luis Zegarra. Asimismo, a Lucas Mísseri, Cristián Fatauros, Luciana Samamé, Emanuel Olivares, Adriana Vercellone, Ignacio Gómez Perdiguero, Ercilia AdÉn y a todo el grupo del Programa en Ética y Teoría Política del Centro de Investigaciones Jurídicas y Sociales (UNC) por sus valiosos comentarios. 


\section{INTRODUCCIÓN}

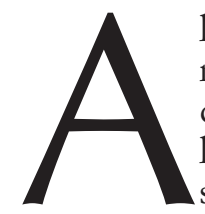

lgunos escritores sostienen que la corrección o incorrección de las acciones se deriva de la bondad o maldad de sus resultados. Como esta afirmación es polémica, haré la siguiente que es, creo, menos controversial: de las acciones que son incorrectas por sus resultados o efectos, muchas lo son por sus efectos adversos sobre personas particulares. De esta clase de acciones, esto es, de las acciones que son incorrectas por sus efectos adversos sobre personas particulares, algunas lo son por el hecho de que estas personas sufren daños a causa de tales acciones. Cuando se afirma que determinadas acciones son incorrectas en situaciones de deforestación de recursos naturales, desecho de residuos en aguas, otras formas de contaminación ambiental, o en procesos de manipulación genética o acciones reproductivas, parece que aquél es el tipo de razonamiento que existe detrás de tal afirmación. En esos temas es natural asumir que dado que es posible dañar a las personas que vivirán en el futuro, tales acciones son incorrectas. De modo que el principio del daño (barm principle) parece una buena herramienta en orden a justificar la censura de tales comportamientos.

Tal principio, utilizado para limitar y justificar moralmente prohibiciones, tiene su origen en los escritos de John Stuart MILL ${ }^{1}$. Mucho se ha debatido, incluso en la época de MiLL, sobre el principio del daño. No obstante, para los fines de este trabajo se puede asumir que el principio del daño es una buena razón para justificar moralmente la censura estatal de ciertas acciones. Es decir que, si tal fuese el caso, no existen reparos morales en la existencia de dicha prohibición.

La versión más ampliamente aceptada y extendida del principio es la propuesta por Joel FEINBERG según la cual «es siempre una buena razón en apoyo de la legislación penal que probablemente sea eficaz para prevenir (eliminar, reducir) un daño a personas diferentes del actor (a quien se dirige la prohibición) y que probablemente no haya otra medida igualmente eficaz a un costo no mayor para otros valores» (FEINBERG, 1984: 26). En este trabajo, no se discutirá su plausibilidad en relación a posiciones rivales que adscriben a ideas populistas morales $^{2}$, perfeccionistas ${ }^{3}$ o paternalistas ${ }^{4}$. El objeto de este trabajo es, meramente, discutir el concepto de daño implicado en el principio del daño.

En la discusión sobre el concepto de daño implicado en el principio del daño FEINBERG afirma que un rasgo distintivo de él es su carácter contra-fáctico. Tal requisito puede darse por cumplido cuando en una situación donde están presente dos sujetos, A y B, «un interés personal de $\mathrm{B}$ está en una peor condición de la que podría haber estado si A no hubiese actuado como lo hizo» (FEINBERG, 1994: 7). A las apro-

1 Quizás la línea más famosa de On Liberty, sea la dedicada a presentar tal principio: «Que la única finalidad por la cual el poder puede, con pleno derecho, ser ejercido sobre un miembro de la comunidad civilizada en contra de su voluntad, es evitar que perjudique a los demás» [MiLL, 1992 (1859): 65].

2 El populismo moral sostiene que para que una acción esté censurada por el estado de modo justificado, sólo es necesario que exista un consenso social respecto de un determinado valor.

3 Una posición es perfeccionista si la ley se utiliza como instrumento para inducir a los individuos a adoptar formas de vida moralmente dignas y a realizar ciertos ideales de perfección humana (NINO, 1980: 289)

4 Para el paternalismo es legítimo utilizar la legislación para ayudar a las personas, aun en contra de su voluntad, a satisfacer lo que se supone que son sus propios planes de vida (NINO, 2013: 127) 
ximaciones que utilizan este elemento para identificar al concepto de daño se las llama tesis comparativa contra-fáctica del daño, o simplemente TCC.

La TCC es ampliamente aceptada y da la respuesta correcta en la mayoría de los casos que es posible considerar. Si A le corta el brazo a B, de modo que el acto de $\mathrm{A}$ hace que B esté en una peor condición de la que estaría si A no le hubiese cortado el brazo, entonces A daña a B. Asimismo, permite explicar el daño ocasionado por ciertas acciones en determinados casos de responsabilidad hacia personas futuras ${ }^{5}$. Por ejemplo, imagínese a un sujeto A que coloca una bomba de relojería en un hospital maternal para que explote dentro de diez meses. Como esto es así, algunas de las personas que estén en el centro de salud cuando detone el artefacto aún no han sido siquiera concebidas. La bomba explota y como consecuencia muchos recién nacidos son heridos ${ }^{6}$. En este caso que involucra a personas futuras — dado que cuando se realiza la acción muchos de los afectados no han sido concebidos-, la TCC puede explicar el daño sufrido por los niños. Si A no hubiese colocado la bomba, los niños no habrían sido heridos y, por tanto, estarían en una mejor condición. El acto de A los ha dañado porque ha hecho que ellos estén en una peor condición de la que podrían haber estado si A no hubiese actuado como lo hizo.

Sin embargo, la TCC encuentra serios problemas en otro tipo de casos de daños a personas futuras. Muestra de ellos son los conocidos ejemplos de PARFIT (1984) ${ }^{7}$ referidos al problema de la no-identidad ${ }^{8}$. Problema así denominado por él, dado que, de acuerdo a cuál sea la acción que se realice, la identidad de la persona resultante será diferente. Imagínese el siguiente caso:

(Moni y Paola): una mujer, Moni, decide concebir a un niño. Ella concurre al médico para realizarse un chequeo. El médico le dice a Moni que si concibe ahora, su hijo tendrá una discapacidad. Tal discapacidad tendrá un impacto negativo en la calidad de vida del niño, aunque sin embargo no es tan grave como para hacer de su vida una tan mala que no valga la pena ser vivida ${ }^{9}$. Supóngase, por ejemplo, que el niño quedará ciego a la edad de diez años. Sin embargo, el médico le comenta a Moni que puede prevenir tal discapacidad tomando una píldora todos los días durante los próximos dos meses. Si ella lo hace, y concibe dentro de dos meses, su hijo será perfectamente saludable. Moni piensa que tomar la píldora por dos meses es ligeramente inconveniente para ella, por lo que decide concebir ahora, y por tanto, sin haber tomado la medicación. Como resultado nace Paola quien queda ciega a la edad de diez años ${ }^{10}$.

La mayoría de las personas ${ }^{11}$ (aunque no todas) ${ }^{12}$ piensa que la acción de Moni de concebir ahora es moralmente incorrecta. En este sentido, dado que Paola quedará

5 Como ha hecho notar Samuel SHEFFLER debe distinguirse el término persona futura de generación futura. El segundo término hace referencia a un colectivo, en tanto que el primero a individuos («Why we Worry about Future Generations?», Lecture in the VI Meeting in Ethics and Political Philosophy, Braga, 8 de junio de 2015). En rigor, en este trabajo se harán consideraciones sobre personas futuras más que sobre generaciones futuras.

6 He modificado el ejemplo de FEINBERG (1984: 97)

7 Quizás los más famosos sean el de la joven de catorce años (PARFIT, 1984: 357) y el de la política arriesgada (PARFIT, 1984: 371).

8 El problema ha sido descubierto casi simultáneamente por Derek PARFIT (1976), Robert ADAMS (1979) y Thomas SCHWARTZ (1979).

9 Para quienes piensan que toda vida es una vida que vale la pena ser vivida, este segundo rasgo puede ser ignorado.

10 He modificado el ejemplo de BoOnIN (2014: 2).

11 BROCK (1995); GOSSERIES (2008); REIMAN (2007); WOODWARD (1986 y 1987).

12 BoOnin (2014: 188-192); Schwartz (1979: 185-186). 
ciega a la edad de diez años debido a la acción de Moni, uno podría pensar que la incorrección moral de tal acción se deriva del hecho de que Paola ha sido dañada. ¿Es esto realmente así? Según la TCC, en el caso en cuestión, esto implicaría que el acto de concebir de Moni ha hecho que Paola esté en una peor condición de la que podría haber estado si Moni no hubiese actuado como lo hizo. Efectivamente, el acto de Moni ocasionó que Paola quede ciega a la edad de diez años. Pero, cla ha dañado? Si se somete el accionar de ella al test contra-fáctico utilizado por la TCC la respuesta es negativa. Paola no está en una peor condición de la que podría estar si Moni no hubiera actuado como lo hizo. Si Moni hubiese tomado la píldora durante dos meses y luego hubiese concebido, Paola no estaría en una mejor condición. Sencillamente no existiría ${ }^{13}$.

Al observar este hecho, muchos han optado por pensar que la objeción moral que podría justificar una censura no puede apelar al daño implicado en el principio del daño ${ }^{14}$. En este trabajo se intentará analizar si una reformulación del concepto de daño implicado en el principio del daño puede explicar la objeción al obrar de Moni y justificar moralmente así la censura de tal clase de acciones.

El problema se puede presentar ayudándose del siguiente silogismo:

P1: Si el acto de $A$ hace que $B$ esté en una condición peor que en la que podría haber estado si A no hubiese actuado como lo hizo, entonces el acto de A daña a B.

P2: El acto de Moni de concebir ahora en lugar de tomar la píldora por dos meses antes de concebir no hace que Paola esté peor de lo que podría estar si Moni no hubiese actuado como lo hizo.

C: Por consiguiente, el acto de Moni no daña a Paola.

Para evitar la conclusión (C) o se debe rechazar P1 o se debe rechazar P2. Dado que cada persona surge de un óvulo y un espermatozoide concreto, y que al cabo de dos meses ni uno ni el otro serán el mismo, Paola no podría haber nacido sin su condición. Podría haber nacido, en su lugar, otro niño al que para propósitos ilustrativos se llamará Coki. Por consiguiente, P2 parece difícil de negar.

De modo que si lo que se quiere evitar es la CE en términos del daño a los seres futuros debe encontrarse una argumento que niegue la P1. Sin embargo, no cualquier negación de tal premisa será satisfactoria. Para ello, el argumento — siguiendo a BOONIN - debe satisfacer tres requisitos: (1) independencia; (2) robustez, y (3) modestia (2014: 19-23). El fundamento de proponer estos requisitos está dado por encontrar la mejor conceptualización posible del daño implicado en el principio del daño que sea capaz de regular y orientar nuestras acciones. De esta manera la tesis que se proponga para resolver el problema debe ser aquella que, comparativamente con otras tesis competentes, aporte mejores resultados en la mayoría de los casos que sea posible considerar.

13 Esto es así porque, independientemente de cuál sea la teoría de la identidad personal que se sostenga, lo cierto es que cada persona surge de un óvulo y un espermatozoide concreto. Como en el espacio de dos meses de concepción, no sólo el espermatozoide sino también el óvulo hubiese sido diferente, es cierto que la persona que — de hecho- fue concebida en otro momento temporal, nunca habría existido. Derek PARFIT denomina a este argumento Tesis de la Dependencia Temporal II: si una persona particular no hubiese sido concebida en el espacio de un mes alrededor del momento en que de hecho fue concebida, de hecho, nunca habría existido (1984: 355). Para quién no esté convencido con tal argumento, vid. con mayor detalle PARFIT, (1984: 351-355) y BOONIN (2014: 29-51).

14 Feinberg (1988: 328); Hurley y Weinberg (2014: 1-24). 
El requisito de la independencia (1), implica que la razón para rechazar la P1 debe ser independiente del hecho de que rechazar la P1 es lo que permite evitar la CE. Pareciera que la existencia de un solo caso donde un principio no pueda dar una respuesta satisfactoria no es suficiente para rechazarlo, si permite solucionar la mayoría de casos que otros principios que compiten con él no pueden. La idea detrás de este requisito es que el concepto de daño que se proponga sea superior al esquematizado en la premisa a rechazar. El requisito de la robustez (2) implica que la razón para rechazar P1 debe ser una buena razón para rechazar también la conclusión evitable (CE) por sí misma. Esto es importante porque muchas soluciones al problema de la no identidad sólo pueden funcionar a costa de solucionar alguna versión cualificada de él, y no la conclusión en sí misma. El objetivo de este requisito es que la tesis propuesta sea lo suficientemente buena como para poder evitar la conclusión a la que no se quiere llegar. Por último, el requisito de la modestia (3) implica que la razón para rechazar la CE no debe generar situaciones o problemas respecto de los cuales se tengan incluso más razones para evitar que las que se tienen para impedir la conclusión que queremos evitar. Si la tesis propuesta genera situaciones aún más contra-intuitivas que aquella presentada en la conclusión, entonces no será una buena tesis.

El análisis se realizará del siguiente modo. En primer lugar, se explorarán diferentes versiones de explicaciones de dañar a otro, basadas en estados. Estas explicaciones se denominan así porque para ellas dañar a otro es colocar a una persona en cierto tipo de estado o condición. De modo que el estatus de daño deriva de lo malo de ese estado final (HANSER, 2008: 421). Dentro de esta explicación se analizarán tanto las ventajas como las desventajas de diferentes tipos de posiciones comparativas y no comparativas. En segundo lugar, se explorará una solución que parece superar los defectos de las posiciones anteriores. Esta tesis se ha denominado como basada en eventos, dado que dañar a otro no implica colocar a la víctima en ninguna clase de estado final, sino en generar cierto tipo de evento. Esta posición aunque prometedora, encontrará ciertas objeciones. Por esta razón se intentará encontrar una conceptualización del concepto de daño implicado en el principio del daño que sea superadora de las anteriores. Finalmente, se analizará si la solución propuesta da cuenta de los tres requisitos exigidos —independencia, robustez y modestia — para resolver el problema de la no identidad.

\section{DAÑAR A OTRO: APROXIMACIONES BASADAS EN ESTADOS}

\subsection{Comparativas}

La tesis comparativa del daño más ampliamente defendida, como hemos dicho, es la contra-fáctica (TCC):

TCC: Si el acto de A hace que B esté en una peor condición de la que podría haber estado si A no hubiese actuado como lo hizo, entonces el acto de A daña a B.

En referencia al problema de la no-identidad, se ha visto en el ejemplo que, dado que la acción de Moni de concebir a Paola no la coloca en una peor condición de la que podría haber estado si Moni no hubiese actuado como lo hizo, entonces el acto de Moni no daña a Paola. Este caso muestra que la TCC no puede explicar por qué se 
piensa que en tal clase de actos se daña a otros. Sin embargo, uno podría argumentar que la TCC sólo falla cuando se aplica al problema de la no identidad. Que, en cambio, en el resto de los casos proporciona una buena explicación, tal y como lo muestran los casos - ya vistos - donde un sujeto le corta el brazo a otro, o aquel donde alguien coloca una bomba de relojería en un hospital maternal. Si esto fuera así, aunque existirían razones para rechazar la TCC, éstas serían más débiles dado que estarían fundadas sólo sobre la base de su imposibilidad para resolver el problema de la no-identidad. De tal manera, la pregunta a considerar es si la TCC puede resolver otras clases de casos.

Un rasgo característico de la TCC es que evalúa el daño sufrido por la víctima teniendo en cuenta su estado final global. Sólo habrá daño si la condición en la que está el sujeto pasivo luego de la acción del agente es peor «en el balance» (on balance) que su alternativa (FEINBERG, 1984: 98 y 1988: 26). Este rasgo es común en varios autores. PARFIT, por ejemplo apela a lo que él denomina tesis C6: «Un acto beneficia a alguien si su consecuencia es que alguien es beneficiado más. Un acto perjudica a alguien si su consecuencia es que alguien es dañado más» (PARFIT, 1984:69). Esta característica, aunque parece beneficiosa en alguna clase de casos, hace que la TCC encuentre problemas en ciertas situaciones que cualquier explicación de lo que significa dañar a otro debería poder solucionar:

(Los lingotes de oro) Imagine a un personaje adinerado - Rodrigo- que vive en una isla. Él está ansioso por un proyecto: desea otorgar algo de su dinero a los vecinos de la isla adyacente. Desafortunadamente, por circunstancias históricas, no se permite a los miembros de la isla donde vive Rodrigo visitar a la otra. Por lo que, para implementar su proyecto, él decide rentar un avión y arrojar lingotes de oro mientras vuela, cada uno de los cuales tiene un valor de diez millones de pesos. Una persona, lamentablemente, es golpeada por un lingote. El impacto quiebra su brazo ${ }^{15}$.

El hombre desafortunado puede curar su herida por mucho menos que diez millones de pesos. De modo que puede, por ejemplo, usar el resto del dinero para mejorar su casa o irse de viaje. A final de cuentas él está en una mejor posición de la que podría estar si Rodrigo no hubiese actuado como lo hizo. Si Rodrigo no hubiese sobrevolado la isla, ni arrojado los lingotes, uno de ellos no hubiese caído sobre su brazo, y él estaría peor. Siendo esto así, ¿ha sido dañado el personaje (des)afortunado? La TCC en la versión que se viene analizando debe responder de manera negativa a tal pregunta. Sin embargo, la intuición indica que Rodrigo ha dañado al personaje desafortunado. Si esto es lo que pensamos, no estamos forzados aún a abandonar las explicaciones comparativas contra-fácticas de dañar. La TCC puede ser reformulada:

TCC2: Si el acto de A hace que B esté en algún aspecto en una peor condición, de lo que podría haber estado si A no hubiese actuado como lo hizo, entonces el acto de A daña a B.

La palabra clave en TCC2 es «en algún aspecto». De esta forma uno podría sostener, de manera plausible, que Rodrigo ha dañado al personaje desafortunado cuando ha arrojado el lingote de oro que le quebró el brazo, dado que «en ese aspecto» lo ha dejado peor de lo que podría estar si el agente hubiese actuado de otro modo, por más que la víctima esté «en el balance» en una mejor condición luego del hecho. Si la

\footnotetext{
15 He modificado levemente el ejemplo de SHIFFrIN (1999: 127).
} 
TCC2 es correcta no hay obstáculos en afirmar que el personaje (des)afortunado ha sido dañado. Después de todo él está «en algún aspecto» en una peor condición de la que podría haber estado de otro modo.

Al igual que en el caso de los lingotes de oro, en el problema de la no-identidad una acción genera dos consecuencias en un ser. Una buena: esto es, haber nacido y tener una vida que vale la pena ser vivida. Y una consecuencia mala: tener una discapacidad. Este dato podría hacer pensar que la acción de Moni ha dañado a Paola dado que parece que en un aspecto ella está peor de lo que podría haber estado si Moni no hubiese actuado como lo hizo, por más que Paola, en el balance, esté mejor. Sin embargo, aunque no se tenga en cuenta la condición de Paola «en el balance», sino sólo en un aspecto de su ser, ella nunca podría haber estado, en ese aspecto, mejor de lo que está. Si Moni no hubiese concebido en el momento en que lo hizo, Paola no tendría el sentido de la vista, porque ella no existiría. Por tanto, conforme a la TCC2 tampoco ha sido dañada.

Sin embargo, como ya se ha afirmado, el problema de la no-identidad no puede ser el argumento decisivo para rechazar una tesis. Si así fuera, no se estaría cumpliendo con el requisito de la independencia. De modo que es necesario analizar otra serie de casos. La TCC2 (y la TCC también) encuentra dificultades a la hora de explicar ciertos casos de daños previos ${ }^{16}$ :

(El matón y el usurero) Supóngase que un criminal quiere robar la tienda de joyas de Joaquín. Dado que el robo será más fácil si Joaquín no está en la tienda, el criminal contrata al matón Ramiro para que le quiebre las piernas a Joaquín el día anterior al robo. Cuando el matón Ramiro llega a la casa de Joaquín, sin embargo, se encuentra con que el usurero local ya le ha roto las piernas a Joaquín ${ }^{17}$.

La TCC2 (y la TCC) no puede explicar de manera convincente el daño sufrido por Joaquín. Tampoco, por tanto, que el usurero lo ha dañado. Según la TCC2: si el acto de $A$ hace que $B$ esté en algún aspecto en una peor condición, de la que podría haber estado si A no hubiese actuado como lo hizo, entonces el acto de A daña a B. En el caso en cuestión, por tanto, siguiendo la TCC2 se debería concluir que nadie ha dañado a Joaquín. Si el usurero no le hubiese roto las piernas, Ramiro lo hubiese hecho. De modo que el acto del usurero no hace que Joaquín esté en algún aspecto en una peor condición de la que de otro modo podría haber estado. El mismo razonamiento se aplica al análisis del matón Ramiro. Ésta es otra razón para rechazar la TCC2 (y la TCC).

La TCC2 (y la TCC) también encuentra problemas cuando existe, en el mismo aspecto del sujeto pasivo, una diferencia temporal entre los efectos positivos y negativos de una acción. Imagínese el siguiente caso:

16 Se llaman daños previos porque su ocurrencia es previa a la de un daño equivalente. Estos casos suelen conocerse como situaciones de sobre-determinación pero, como reconoce HANSER, esta denominación no es del todo correcta ya que algo está sobre-determinado si es el resultado de la operación conjunta de dos o más causas, cada una de las cuales ha sido condición suficiente para la producción del evento por su propia cuenta (HANSER, 2008: 436). En la literatura penal continental se llama a la primer clase de casos como de cursos causales hipotéticos, en tanto que los segundos como de cursos causales alternativos. En los casos de sobre-determinación, creo que la única solución es entender a las diferentes acciones de las personas como condiciones suficientes y no como condiciones necesarias. De modo que si se constata un determinado resultado cualquiera que haya puesto una condición suficiente para que ocurra lo habrá causado (vid. PARFIT, 1988: 18).

17 He modificado ligeramente el ejemplo de HANSER (2008: 434). 
(El Murciélago Negro) Tony es atacado con ácido por Snate y queda ciego. Un cirujano realiza un trasplante de corneas en Tony. Cuatro meses más tarde le retiran las vendas y Tony descubre que no sólo puede volver a ver normalmente, sino que además puede ver en plena oscuridad. De esta manera Tony está luego de un tiempo mucho mejor de lo que estaba incluso antes de que le hayan arrojado ácido ${ }^{18}$.

Una aproximación como TCC2 debería afirmar que Tony no ha sido dañado ya que no está, a final de cuentas, peor en un aspecto de lo que estaría de otro modo. Un resultado similar se alcanza desde la TCC dado que Snate estará «en el balance» mejor. Tales conclusiones parecen implausibles. Esto es una nueva razón para rechazar a ambas versiones de TCC.

Existen otras situaciones donde las explicaciones contra-fácticas tienen dificultades para dar una respuesta satisfactoria. Cuando alguien muere a consecuencia de la acción de otro, no hay ningún nivel de bienestar (o de lo que sea que haga que una vida valga la pena ser vivida) para el sujeto que muere que sea menor de lo que sería de otro modo. Si el estatus de daño deriva de lo malo del estado en el que se está, el estado de «estar muerto» no tiene valor para la persona: ni positivo, ni negativo, ni neutral (HANSER, 2008: 437). Desde que esto es así, entonces, no hay posibilidad de hacer comparación posible y, por tanto, resulta difícil explicar el daño en estas situaciones.

Después de lo expuesto, parece que la TCC en cualquiera de sus dos versiones debe ser rechazada. ¿Es esto una buena noticia para resolver el problema de la no identidad? Recuérdese que en el caso de Moni, la premisa a negar es P1 ${ }^{19}$. P1 es lo que implica (aproximadamente) cualquier versión de la TCC. Como cualquier versión de la TCC es falsa, P1 debe ser falsa, pero ¿es lo suficientemente falsa como para hacer desaparecer el problema de la no identidad? Si la respuesta es negativa no se habrá podido evitar la conclusión que queremos evitar, dado que el argumento no es tan robusto como para negarla por sí misma. La única manera de cumplir con tal requisito sería encontrar algún sustituto para P1 que implique que el acto de Moni daña a Paola. Dentro de las explicaciones de dañar a otro fundadas en estados, las posibilidades parecen ser dos: o bien se reemplaza la línea de base sobre la cual se realiza la comparación ${ }^{20}$; o bien se abandona la explicación comparativa.

La primera alternativa no parece muy prometedora. Se han propuesto dos líneas de base diferentes respecto de las cuales hacer la comparación. De acuerdo a la primera, si el acto de alguien hace que otro esté en una condición que es peor a la que estaba antes, entonces ese acto lo ha dañado. Esta tesis, seguida (parcialmente) por importantes filósofos ${ }^{21}$ no puede explicar por qué Moni daña a Paola, sencillamente porque Paola no está antes de la acción de Moni en ninguna condición, y por tanto no existe condición que pueda empeorar. Piénsese por ejemplo que, todo lo demás igual, Paola en vez de perder la vista a la edad de diez años, ella nace ya en esa condición

18 He modificado el ejemplo de HANSER (2008: 424).

19 P1: Si el acto de A hace que B esté en una condición peor que en la que podría haber estado si A no hubiese actuado como lo hizo, entonces el acto de A daña a B.

20 Lo que suele conocerse como el problema de la línea de base del daño (PETERSEN, 2014: 3 ).

21 Joseph RAZ sostiene «uno daña a otro cuando la acción de alguien hace que las personas estén peor de lo que estaban antes, o de lo que tienen derecho a estar, de modo que afecte a su bienestar futuro» (1986: 414). Un argumento similar puede verse en RAZ (1987: 329). 
(ciega). Dado que ella nace en esa condición, y por tanto no empeora, no parece que esta clase de tesis pueda explicar de manera satisfactoria el daño sufrido por Paola. Del mismo modo, tales clases de tesis tienen inconvenientes en todos aquellos casos no atravesados por el problema de la no-identidad donde el sujeto que se afecta no está peor luego de la acción del agente ${ }^{22}$, o en aquellos en los que está peor, pero mejor de lo que estaría de otro modo ${ }^{23}$.

La segunda forma de reformular la línea de base ha sido denominada como maximizadora. De acuerdo a esta aproximación, «un acto daña a una persona, si tal acto crea menos bienestar para esa persona, cuando el agente —o grupo de agentes - tenía la alternativa de crear más» (ROBERTS, 2009: 203). Supóngase que una persona le dispara a otra en el brazo en lugar de hacerlo en el corazón. Pareciera que conforma a cualquier versión de la TCC, el agente no ha dañado a la víctima, dado que si no hubiese actuado como lo hizo, la víctima estaría peor. En cambio, si se sigue una aproximación maximizadora es posible explicar por qué el agente ha dañado a la víctima. Esto es así porque para aquel existe un curso de acción disponible donde produciría que la víctima se encuentre en una mejor condición que lo que estaría en aquellas que tuvo en cuenta ${ }^{24}$.

Aunque esta idea parezca plausible, aún requiere ciertas precisiones. En primer lugar, obviamente, para evaluar los cursos de acción disponibles se deben eliminar aquellos cursos de acción que sean inaccesibles para el agente al tiempo de actuar, ya sea porque estén bloqueados por alguna ley de la naturaleza, o por actos de otras personas que escapen a su control (ROBERTS, 2007: 277). Nadie puede ser responsable por el daño que alguien sufre cuando un rayo en una tormenta lo ha matado. Incluso si el agente, como en el conocido ejemplo, ha enviado a su tío rico a caminar por el bosque durante una tormenta con la esperanza de que un rayo caiga sobre él, para heredarlo. En segundo lugar, para evaluar el daño no sólo deben tenerse en cuenta las restricciones naturales o aquellas derivadas de los actos de otros, sino también las epistémicas. El entendimiento del daño de esta manera requiere que el agente realice un cálculo de probabilidades respecto de las consecuencias de las diferentes opciones que tiene para actuar. Esta tesis parece prometedora pero tiene un defecto: confunde dos aspectos involucrados en la temática: el concepto de daño y el de reproche por el daño causado. El análisis de ROBERTS es una buena explicación — aunque requiere ciertos ajustesde cuándo uno debe ser responsable por algún acto, no respecto de lo que significa dañar a otro. Pero un análisis de este asunto escapa a los límites de este trabajo ${ }^{25}$.

22 Un caso de tal tipo sería el siguiente: (Paraplejia genética) Supóngase que Marcela, una especialista en genética es doctora en una clínica de fertilidad. Ella fecunda cierto óvulo O con el Esperma E, de manera que cuando sea implantado, el resultado será que una persona tendrá paraplejia genética, es decir el estado que consiste en estar genéticamente programado para nacer parapléjico.

23 Por ejemplo, en: (El Parquímetro) El parquímetro donde el automóvil de Pablo está estacionado ha expirado y el oficial de policía está por descubrirlo. Si él lo hace, le impondrá una multa por 50\$. Justo antes de que el oficial de tráfico descubriera la infracción de Pablo, una amiga suya — Romina— que se encontraba caminando por el lugar ve la situación. Romina no tiene dinero en su billetera, pero observa que Pablo ha dejado la puerta abierta y que tiene unas monedas sobre el asiento. Ella toma dos pesos de su auto y lo coloca en el parquímetro. Como resultado, Pablo tiene dos pesos menos en su auto, y no tiene que pagar la multa de $\$ 50$.

24 Esta tesis podría ser sintetizada así: si el acto de A hace que B esté en una condición que es peor que aquélla en la que A lo podría haber colocado, entonces A daña a B.

${ }_{25}$ Para un análisis detallado de ésta y otras tesis comparativas vid. S. TrucCONE BorgOGNO, «Entre la Utilidad y el Daño: el problema de la no-identidad», Tèlos: Revista Iberoamericana de Estudios Utilitaristas (en revisión). 
Luego de este análisis, parece que ninguna aproximación comparativa puede dar una respuesta satisfactoria al problema de la no-identidad. La razón sería que —en nuestro ejemplo_- Paola no puede estar peor que ninguna línea de base posible.

\subsection{No comparativas}

La segunda alternativa, entonces, es abandonar las tesis comparativas y pasar a las no-comparativas. En líneas generales, las tesis no comparativas consideran que se ha dañado a alguien cuando se causa que una persona esté en un estado malo ( $b a d$ ) (o tenga una condición mala). La idea es que existen ciertos estados que tienen determinadas propiedades que son intrínsecamente malas. Así, cualquier persona que estuviera en tales estados estaría sufriendo un daño. Por tanto, cualquiera que cause que otro esté en un estado tal que signifique que está sufriendo un daño, lo habrá dañado. Por ejemplo, el hecho de tener un brazo cortado es algo malo. Así, cualquiera que esté en ese estado, según esta tesis, estaría sufriendo un daño. De manera que cualquiera que haya causado que la «víctima» esté en ese estado habrá dañado. Del mismo modo, ser ciego es un estado que tiene propiedades intrínsecamente malas. SI alguien está en ese estado, estará sufriendo un daño. De modo que si alguien ha causado que otro esté en ese estado, lo habría dañado. La tesis no comparativa de dañar, o simplemente TNC, podría sintetizarse como sigue:

TNC: Si el acto de A causa que B esté en un estado malo, entonces A daña a B.

La ventaja de esta clase de aproximaciones, frente a las comparativas, es que alcanza con afirmar que alguien ha colocado a otra persona en un estado malo para decir que la ha dañado. De tal manera, los problemas presentados por las tesis comparativas respecto de — por ejemplo- los daños previos o la diferencia temporal en los efectos positivos y negativos de una acción, aquí no tendrían lugar. En el primero de los casos, que se ha ejemplificado con «el matón y el usurero local», las tesis contra-fácticas no podían dar una respuesta totalmente satisfactoria, debido a que la víctima quedaba en el mismo estado final, tanto si el usurero le quebraba la pierna a la víctima o no. Las tesis no-comparativas, por su parte, no encuentran inconvenientes en esta clase de casos ya que, para ellas, dañar a otro consiste en colocar a la víctima en un estado malo (no-comparativo y absoluto). Si esto es así, el usurero que quiebra las piernas de la víctima, la daña porque la coloca en ese estado malo. Sin importar si hubiese estado en esa condición de todos modos.

Las tesis no-comparativas también pueden resolver casos como el del Murciélago Negro, donde un evento coloca a un sujeto en un aspecto peor por un tiempo, pero luego la víctima está mucho mejor que incluso antes, en ese mismo aspecto. Las tesis no comparativas sólo necesitan afirmar que el agente ha colocado a otra persona en un estado con propiedades intrínsecamente malas. Como la víctima está, en un momento, en un estado de esa naturaleza, entonces ella habrá sufrido un daño. Cualquiera que haya causado ese estado de cosas en un sujeto, lo habrá dañado. Como las TNC pueden resolver gran cantidad de casos que las tesis comparativas encuentran dificultosos, son candidatas a ser superiores y - por tanto- a resolver el problema de la no-identidad. 
Sin embargo, esta clase de tesis, también encuentra algunos inconvenientes en distintas clases de casos. Les es muy dificultoso explicar por qué la muerte es un daño para alguien. Según las construcciones no-comparativas, sufrir un daño es estar en un estado malo - es haber sido colocado en un estado de cosas que es malo para el que lo sufre- - Ahora bien, si lo malo deriva del estado en el que se está, dado que «estar muerto» no es ni bueno, ni malo, ni neutral, es difícil observar cómo esta clase de tesis puede acomodarse en tales casos. Parece que, por tanto, según las TNC estar muerto no es sufrir un daño. Si esto es así, no parece posible sostener que causar que alguien esté en un estado que no implica sufrir daño, ha causado un daño.

Otro inconveniente para las TNC tiene lugar en situaciones donde determinada acción hace que las personas estén peor de lo que estarían de otro modo, pero en estados que no tienen ninguna propiedad intrínsecamente mala. Imagínese el siguiente caso:

(El ganador del premio Rolf Schock): Tim ha golpeado en la cabeza a Derek, ganador del premio Rolf Schock en filosofía y lógica. Como resultado, Derek tiene ahora una inteligencia promedio y sus trabajos académicos han bajado de nivel ${ }^{26}$.

¿Ha dañado Tim a Derek? La respuesta parece ser positiva. Sin embargo, hacer tal afirmación es sumamente dificultoso a partir de las tesis no-comparativas. Un estado donde se tenga una inteligencia promedio no parece tener alguna propiedad intrínsecamente mala. Si esto es así, la pregunta es ¿por qué colocar a alguien en un estado que no tiene propiedades intrínsecamente malas (es decir, un estado que no implica sufrir un daño) es dañarla? Las tesis no comparativas encuentran serias dificultades a la hora de resolver esta clase de casos. Derek no está en ningún estado no-comparativamente malo. Y, por tanto, desde esta tesis no puede ser un daño colocarlo en ese estado.

Aunque esto sea así, ciertas versiones no comparativas de la tesis del daño, como la de SHIFFrin (1999 y 2012) pueden resolver el caso bajo análisis. Según su posición, si el acto de $\mathrm{A}$ hace que $\mathrm{B}$ esté en un estado en el cual B no querría racionalmente estar, entonces $\mathrm{A}$ daña a $\mathrm{B}$. La idea detrás de esta tesis es que para saber si alguien sufre un daño, debemos preguntarnos si él consentiría en que le ocurra lo que le paso. Siendo esto así, parece que Derek no podría consentir en tener una inteligencia promedio, de modo que —entonces - Tim habría dañado a Derek. Sin embargo, esta solución paga el precio de generar un nuevo problema. Esto es, no poder explicar situaciones donde los sujetos pierden ciertos bienes y ellos no se oponen a tal situación (HANSER, 2008: 432). Imagínese que el golpe que Tim le propina a Derek, en lugar de dejarlo con poderes intelectuales promedio, hace que olvide su vida pasada, y habiéndola olvidado él está feliz porque ha incrementado su trabajo intelectual (THOMSON, 2011: 440). Si nos preguntamos si Derek consentiría lo sucedido, la respuesta parece ser positiva, Derek no se opone a lo que le sucedió. Sin embargo, parece que él, dado que recibió un golpe en la cabeza y olvido su vida pasada, está en un estado no-comparativamente malo, y por tanto ha sido dañado. Para resolver este caso, SHIFFrin debería abandonar el entendimiento de sufrir un daño como de estar en estados en los que no se querría

26 He modificado el ejemplo de HANSER (2008: 432). 
racionalmente estar. Pero si esto es así, no podría explicar por qué Derek ha sido dañado en el primer caso.

Existe una dificultad más general en referencia a las tesis no-comparativas del daño. El problema está dado por encontrar un criterio que pueda unificar o al menos identificar a diferentes estados a los que llamamos daño. Tales tesis, a excepción de la propuesta de SHIFFRIN, parecen dejar librado a la intuición qué clase de estados deben considerarse como malos. Algunos hablan de umbral de normalidad (RIVERA LÓPEZ, 2011: 100), otros simplemente enumeran ciertos estados malos tales como la enfermedad, la deformidad, una discapacidad, molestia física o la muerte (HARMAN, 2009: 139). Si tal tesis ha de convencernos para que sea utilizada en cuestiones prácticas, debería decir más respecto de ese punto.

Asimismo, todas esas posiciones, incluyendo la de SHIFFRIN, se encuentran con el siguiente problema, que ha sido puntualizado por A. SMAJDOR (2014: 6). Si se asume que los estados en los cuales pueden estar las personas van de digamos 1 a 1.000 puntos, donde cada punto representa una unidad de sufrimiento, siendo 1000 la muerte. Uno podría suponer que el umbral el daño puede estar marcado por los 800 puntos. Supóngase que alguien realiza una acción que tiene consecuencias en tres personas diferentes. En la persona A la coloca en un estado de 800 puntos; a la persona B en un estado de 799 puntos y a la persona $C$ en un estado de 1 punto. De acuerdo a las tesis no-comparativas sólo $\mathrm{A}$ ha sido dañada, por más que la diferencia con $\mathrm{B}$ es imperceptible. Del mismo modo, si se compara B con C parece que la diferencia es enorme. Sin embargo, tal tesis debe afirmar que B no ha sido dañada. Esto parece implausible. Algunos autores responden a esta objeción afirmando que podrían entenderse a los estados malos o al umbral no como una línea, sino como un espacio, de manera que se permita cierto margen de variación (RIVERA LÓPEZ, 2011: 119). Esta réplica le quita fuerza a la objeción, aunque — creo—, no la elimina completamente. Llegará algún punto en el cual el espacio no puede «ensancharse» más, y se replicará el inconveniente.

Dadas las dificultades de las tesis no-comparativas, parece que no han demostrado ser superiores a las comparativas. Sin embargo, tampoco han quedado en desventaja respecto de aquellas, ya que pueden resolver algunos casos que las comparativas no. De modo que, hasta el momento no hay razones para preferir a una sobre la otra. Lo único que se sabe es que las dos tienen problemas en diferentes clases de casos. Por tal razón, aunque no sea una explicación completa de lo que implica dañar a otro, tal vez pueda resolver el problema de la no-identidad.

Conforme a las TNC parece posible explicar por qué el acto de Moni daña a Paola. Esto es así porque desde que ser ciego es estar en un estado malo y Paola es ciega, dado que Moni causó tal estado, entonces, ella la ha dañado. Sin embargo, existen ciertos inconvenientes vinculados a esta aproximación para explicar por qué Moni ha dañado a Paola. El problema surge al comparar tal caso con aquellos donde el agente causa que alguien esté en un estado malo, pero no tan malo como el estado en el que podría haber estado si el acto no se hubiese realizado:

(El cirujano) Has tenido un grave accidente que te deja inconsciente. Tan grave que si un cirujano no realiza un procedimiento extremo, morirás. Por tanto, el cirujano amputa 
tu brazo porque es la única manera de salvar tu vida. Lo que el cirujano hace es mejor que cualquier posible alternativa. ¿Te ha dañado al amputar tu brazo? ${ }^{27}$.

Un defensor de la tesis no-comparativa debería mostrar cómo es posible defender la afirmación de que el médico no te ha dañado, a la vez que la acción de Moni de concebir a Paola la ha dañado a pesar del hecho de que, en ambos casos el agente hace que el sujeto pasivo de la acción no esté peor de lo que podría haber estado de otro modo. Después de todo parece que, como afirma MCMAHAN «si es incorrecto causar que alguien exista con una condición $\mathrm{X}$, entonces debería también ser incorrecto salvar la vida de alguien si el único medio para hacerlo causaría también que la persona tenga una condición X» (1998: 224).

Se han hecho varios intentos en orden a responder a tal pregunta. Los dos más relevantes han sido proporcionados por Elizabeth HARMAN (2004 y 2009) ${ }^{28}$ y Seana SHIFFRIN (1999 y 2012) ${ }^{29}$. En otro lugar he explicado con detalle por qué ellos están condenados al fracaso ${ }^{30}$. Sin embargo, a los fines que aquí interesan basta con apuntar que ambas propuestas tropiezan con una, creo, crítica devastadora. Si el concepto de daño tiene algún sentido, es porque a través de él se pretende proteger a la persona que lo sufre de determinada afectación negativa. La función de calificar a diferentes estados como «malos», porque tienen características intrínsecamente malas, es crear una barrera que deje a las personas en estados diferentes a ellos. Sin embargo, en los casos atravesados por el problema de la no identidad, pareciera que tales estados cumplen una función inversa. La calificación de determinado estado como malo, no hace que la persona que se quería proteger esté mejor que ese estado malo (o esté en un estado bueno), sino sencillamente no exista ${ }^{31}$.

\subsection{Una aproximación combinada}

Dado que tanto las tesis comparativas como las no-comparativas tienen problemas en distintas clases de casos, tal vez la solución sea optar por una tesis combinada. Después de todo, parece que las personas a la hora de identificar un daño, tienen intuiciones comparativas en determinados casos y en otros, intuiciones de tipo no comparativo. Una propuesta de este tipo es la llamada tesis combinada, propuesta por Lukas MEYER (2003, 2009 y 2015) Su propuesta comienza con una afirmación de tipo

27 He modificado el ejemplo de PARFIT (1986: 855).

28 «Una acción daña a alguien si la acción causa dolor, muerte prematura, daño corporal, disconformidad incluso si ella no hubiese existido si la acción no se hubiese realizado» (HARMAN, 2004: 92).

29 «Daño implica condiciones que generan una brecha [chasm] o conflicto importante entre la voluntad y la experiencia de uno, la propia vida entendida en términos más generales, o las circunstancias de cada uno» (SHIFFRIN, 1999: 123).

30 Vid. S. Truccone Borgogno, «¿Puede el no-comparativismo resolver el problema de la no-identidad?», Daimon: revista internacional de filosofía (en edición).

31 Jeff McMAHAN utiliza un argumento similar para descartar las soluciones al problema de la no identidad que apelan a los derechos de las personas a las que los actos afectan. La respuesta anclada en la violación de derechos parece no prestarle demasiada atención a que la persona cuyos derechos han sido violados sólo puede existir, de hecho, en un estado en el cual sus derechos han sido violados (MCMAHAN, 1981: 125). Con todo, aún si este argumento fallase, habría que encontrar otro que explique por qué sería objetable causar que un niño nazca con estados malos, cuando esa era su única alternativa y a la vez tiene una vida que vale la pena ser vivida, algo que puede catalogarse como estado bueno (MCMAHAN, 1998: 216). 
no-comparativo. De acuerdo a ella «una acción daña a una persona si a consecuencia de tal acción la persona cae por debajo de un umbral normativamente definido» (MEYER y ROSER, 2009: 228). Tal entendimiento no es afectado por el problema de la noidentidad dado que para encontrar daño no se requiere una comparación hipotética con la situación que hubiese ocurrido en ausencia de la acción dañina. La idea general detrás de la interpretación del umbral del daño es que se tiene el deber general de no causar que las personas estén en una peor condición que aquella en la que deberían estar (MEYER y ROSER, 2009: 229). La tesis del umbral puede ser resumida como sigue: si el acto de $A$ hace que B caiga debajo de un umbral específico, entonces A daña a B.

Si, por ejemplo, el umbral está marcado por tener un sentido de la vista normal, esta tesis implica que, dado que Moni al concebir a Paola hizo que ella sea ciega, desde que ser ciega cae debajo del umbral, Moni ha dañado a Paola. Como se observa, hasta aquí, esta aproximación es esencialmente no-comparativa y, por tanto parece quedar sujeta a las mismas objeciones que aquellas. De modo que si pretende ser superadora, debería dar una razón diferente a las ya analizadas a los fines de resolver los inconvenientes que se le pueden presentar. El primer problema, como ya se ha puntualizado en otra parte de trabajo, está dado porque tal tesis parece sobre-inclusiva. Es decir que considera como daños a situaciones que no deberían serlo. Si causar un daño es colocar a alguien por debajo de cierto umbral, entonces un médico que mejora la condición de un paciente pero lo deja por debajo de él, lo ha dañado. Esto parece implausible. Sin embargo, MEYER sostiene que, en este caso, el médico no puede evitar dañar a su paciente: haga lo que haga lo dejará por debajo del umbral. Si esto es así, no tiene sentido afirmar que en ese caso existe una obligación de no causar que la persona quede por debajo de cierto umbral, esto es de causar daño. A nadie se le puede exigir que haga aquello que está fuera de sus posibilidades. Sin embargo, sí se les puede exigir que hagan aquello que menor cantidad de daño produzca (MEYER, 2003: 153), es decir, que uno impida que alguien esté en una condición peor por debajo del estándar: si no lo hace, habrá dañado más al sujeto pasivo. Esta respuesta parece satisfactoria, sin embargo, se puede objetar que tal afirmación no está implicada en la tesis del umbral, sino en aproximaciones comparativas. Cuando el autor afirma que existe la obligación de «minimizar el daño» parece utilizar no un concepto no comparativo sino uno comparativo. Si esta tesis ha de convencernos habría que dar una razón para justificar este cambio en el seno de la misma posición.

La segunda objeción a la tesis es que es infra-inclusiva. El caso del ganador del Premio Rolf-Schock ejemplificaba este punto. Si alguien está peor de lo que estaría de otro modo, pero todavía por arriba del umbral, parece que de todos modos ha sido dañado. Aquí, otra vez, una aproximación comparativa da la respuesta correcta en detrimento de la que venimos analizando. Una defensa de esta tesis, nuevamente, puede apelar a la obligación de dejar a la persona a quienes las consecuencias afectan lo más lejos posible del umbral. Parece que, entonces tanto las nociones no comparativas como las comparativas deben tener lugar en nuestro concepto de daño. Si esto es así debería adoptarse una tesis combinada ${ }^{32}$, o TComb (MEYER, 2003: 154-155). De acuerdo con ella:

32 Jeff MCMAHAN, por ejemplo, también considera que es inevitable apoyar una aproximación pluralista o combinada del daño que involucre tanto aspectos comparativos como no-comparativos (McMAHAN, 
TComb: Si A realiza un acto que o

(1) causa que B caiga por debajo de cierto umbral y, si no es posible evitar causar daño en ese sentido, A no minimiza el daño en B; o

(2) causa que B esté peor de lo que estaría si A no hubiese actuado como lo hizo entonces A daña a B.

Pareciera que esta tesis podría resolver toda clase de casos que se puedan presentar. Sin embargo, más allá de esa consecuencia plausible, no se han dado demasiados argumentos para afirmar que adoptar tal conceptualización del daño está justificado. Parece que sería necesaria alguna razón que explique por qué la misma tesis se maneja con dos nociones diferentes de daño. Adicionalmente, el problema con esta aproximación es que, en primer lugar, parece que sólo necesita del umbral para explicar el daño que Moni le causa a Paola, en tanto que para todo otro posible caso utiliza una explicación comparativa. Si esto es así, entonces, no se habría cumplido con el requisito de la independencia. Segundo, aún si este argumento no es contundente. Parece que ni siquiera con la combinación propuesta puede resolverse el problema de la no identidad. Si para tal caso se afirma que alguien ha sido dañado cuando ha sido colocado por debajo de cierto umbral, nuevamente, pareciera que el umbral cumple una función inversa a la que persigue. La idea del umbral es proteger a las personas afectadas por nuestros actos. En el caso de Moni y Paola, el umbral no protege a Paola. Su existencia no hace que ella esté por encima de él, sino que — sencillamente- no exista.

Tercero, dado que se intenta encontrar una tesis del daño que pueda cumplir funciones prácticas, debería poder explicitarse cómo se conforma el umbral, es decir qué es aquello que las personas deberían tener y, en ausencia de lo cual, se puede sostener que han sido dañadas (SMAJDOR, 2014: 14). Asimismo, si para identificar al daño implicado en el principio del daño se debe conocer lo que las personas tienen derecho a tener, entonces la discusión relevante ya no sería en términos del principio del daño, sino en términos de una teoría de la justicia. Es decir, ¿cuál sería el valor del daño si lo que tal concepto pretende resolver ya lo ha hecho una teoría de la justicia? Algunos autores han criticado a los defensores de ciertas versiones del principio del daño por implicar tal conclusión (Holtung, 2002: 385). Prueba de ello es que el mismo MeYER, al momento de tener que especificar el umbral, discute la formulación que de él puede hacerse desde concepciones suficientaristas, igualitaristas o prioritaristas (MEYER, 2015: 13-17). Si esto es así, entonces, el daño y el principio del daño no aportan ninguna razón extra a las que se pueden extraer de la discusión sobre la teoría de la justicia, de manera tal que la polémica sobre el concepto de daño en orden a defender el principio del daño no tendría demasiado sentido. Finalmente, dado que TComb es una teoría basada en estados, tampoco podría explicar por qué la muerte es un daño.

\section{DAÑAR A OTRO: APROXIMACIÓN BASADA EN EVENTOS}

Una alternativa interesante a las tesis que se acaban de analizar es la propuesta por Matthew HANSER. El autor observa, al igual que los partidarios de las tesis com-

2012: 4). Sin embargo, más allá de esta afirmación y la ejemplificación de diferentes casos donde alternativamente uno $\mathrm{u}$ otra posibilidad funcionan, no da demasiados argumentos. 
binadas, que las personas tienen en algunos casos intuiciones comparativas a la hora de afirmar por qué alguien ha sufrido un daño, en tanto que en otros casos parece que con el sólo hecho de que el sujeto esté en un estado malo, es suficiente para afirmar que él sufre un daño. HANSER cree que lo que fundamenta esta clase de intuiciones contradictorias es que cuando las personas piensan en el daño, en realidad no están haciendo referencia al estado final en el que están, sino a eventos que les suceden. El rasgo distintivo de esta explicación es que es una aproximación basada en eventos y no, como las anteriores, una basada en estados. Con arreglo a las tesis basadas en eventos, dañar a otro no implica colocar a la víctima en ninguna clase de estado final, sino en generar cierto tipo de evento. Sufrir un daño, por tanto, será ser objeto de un tipo de evento, independientemente de la maldad o bondad de cualquier estado resultante en que se encuentre la persona que lo sufre (HANSER, 2008: 440).

El argumento de HANSER puede ser reconstruido como sigue. En primer lugar, el autor distingue claramente dos conceptos: sufrir un daño y causar un daño. De esa manera es posible analizar la noción «daño» en dos pasos. Uno de ellos es identificar lo que implica dañar (o causar daño); el otro es explicar cuándo alguien sufre un daño.

Paso uno: A daña a B actuando en cierto sentido si y sólo si $\mathrm{A}$ actuando en tal modo queda en una relación $\mathrm{R}$ con algún daño sufrido por $\mathrm{B}$.

Paso dos: B sufre un daño si y sólo si ___ (HANSER, 2009: 181).

De esta manera, la reconstrucción del argumento de HANSER debe hacerse también en dos pasos. Se debe tratar de explicar qué significa dañar a otro; y qué significa que alguien ha sufrido un daño. Se comenzará con la explicación de lo que significa sufrir un daño. El autor inicia describiendo la noción de «bien básico». Para él, un bien no es un estado o condición en la que sería bueno estar, sino es algo que es bueno tener. «Bienes básicos» serán aquellos cuya posesión hace posible alcanzar una amplia variedad de componentes potenciales de una vida razonablemente feliz (HANSER, 2008: 440). Podría afirmarse que un bien básico es aquello que es necesario para que cada quien pueda gobernar su vida de acuerdo a sus propias decisiones. El sentido de la vista, por ejemplo, puede ser catalogado como un bien básico, de modo que cualquiera que lo pierda estará sufriendo un daño. Así, (1) «Alguien sufre un daño de nivel-1 respecto de cierto bien básico sólo si pierde alguna cantidad de tal bien» (HANSER, 2008: 441). Uno podría decir que si Juan dispara en el brazo a Pedro, de modo que este último tiene su brazo inutilizado por un mes, entonces Pedro ha sufrido un daño de nivel-1 porque ha perdido cierta cantidad del «bien básico» integridad física.

Sin embargo, como el mismo autor puntualiza, no todos los daños consisten en pérdidas de bienes básicos. Si una persona mata al cirujano que iba a devolverle la vista a José, este último ha sido dañado por no recibir ese beneficio. Este daño pertenece a la categoría de los llamados daños preventivos, los cuales consisten en haber impedido que alguien reciba cierta clase de beneficio. Dado que no toda vez que uno sea impedido de recibir un beneficio ha sido dañado, entonces los daños preventivos deben derivar de los daños de nivel-1. En términos de HANSER: (2) «alguien sufre un nivel de daño $(n+1)$ respecto de cierto bien básico si y sólo si se ha visto impedido de recibir un nivel- $n$ de beneficio respecto de un bien básico» (2008: 442) Por tanto (3) «alguien sufre un daño si y sólo si él sufre un daño de algún nivel respecto de un bien básico» (2008: 442). De modo que sufrir un daño implica, o (1) que alguien pierde cierta can- 
tidad de un bien básico; o (2) que a alguien se le haya impedido de adquirir cierta cantidad de un bien básico. Así, cualquiera a quien le haya ocurrido un evento que haya ocasionado en él esta consecuencia habrá sufrido un daño, independientemente de cuál sea su estado resultante.

El segundo aspecto a analizar es, la noción «A daña a B». El centro de la atención deberá estar en qué es lo que significa estar en una relación $\mathrm{R}$ respecto del daño sufrido por alguien. Para esta posición estar en una relación $\mathrm{R}$ respecto del daño sufrido por un sujeto implica haber realizado una acción que causó el evento que hice que la víctima sufra un daño (HANSER, 2009: 185). Así, si sufrir un daño es haber sido objeto de cierto tipo de evento, y dañar a alguien es estar en una relación $\mathrm{R}$ respecto del daño sufrido por alguien, entonces dañar a alguien es haber realizado una acción que causo el evento que produjo el daño en ese sujeto. En suma, la tesis basada en eventos, o TBEv:

TBEv: si el acto de A produce un evento que hace que $B$ pierda o se vea impedido de adquirir cierta cantidad de algún bien básico, entonces $\mathrm{A}$ daña a $\mathrm{B}$.

La ventaja de la tesis de HANSER respecto de las aproximaciones basadas en estados es que no requiere analizar el estado final de la víctima para saber si ha sufrido o no un daño. Por tanto, tal vez pueda resolver los problemas que tenían tanto unas como otras tesis. Respecto de los problemas que suscitan las tesis comparativas, la TBEv pude evitarlos. En los daños previos, la TBEv da la respuesta correcta. Así, en el caso del matón y el usurero, el dueño de la tienda, que ha visto sus piernas rotas, ha perdido cierta cantidad de un bien básico. Cualquiera que haya causado el evento que lo generó, lo habrá dañado. Independientemente de si su estado final hubiese podido ser el mismo —o mejor o peor- por otra vía.

Respecto de la muerte, en general, la TBEv también da —en principio-la respuesta correcta. Morir es un daño, porque se pierde cierta cantidad de un bien básico. Dado que el estatus de daño deriva del evento que se sufre y no de lo malo del estado en el que se está, no importa qué clase de estado es la muerte — si es bueno, malo o neutral- Sin embargo, el problema con esta afirmación es que morir no parece ser siempre algo malo. Si la vida sería aún peor, morir puede ser considerado un beneficio $^{33}$. La TBEv de HANSER parece no permitir hacer esta afirmación. Sin embargo, el autor da cuenta de este aspecto y afirma que la TBEv debe distinguir dos clases de escenarios. El primero sería aquel donde los poderes vitales del sujeto han sido tan disminuidos que no tienen valor para él, de modo que tal vez no merezcan seguir siendo bienes básicos; en tanto que el segundo sería aquel donde los poderes vitales se mantienen intactos (HANSER, 2011: 449). Si esto es así, se estaría en presencia de un daño sólo cuando se prive a un sujeto de la vida en la segunda clase de casos. Esto parece plausible, sin embargo, el problema es que la primera clase de casos parecen ser una excepción a su propia tesis, dado que sugiere que la maldad del evento no depende de él mismo, sino de algo más, en este caso del estado en el que está y del estado en el que pudiera quedar la persona.

En cuanto a los problemas de las tesis no-comparativas, ellos se presentaban sobre todo en casos donde la víctima está en un estado malo pero no tan malo como

\footnotetext{
33 Vid. PARFIT (1984: 489-490).
} 
el que de otra manera podría estar. $\mathrm{O}$ en casos donde la víctima queda en un estado no-comparativamente no malo pero que (comparativamente) es peor de lo que podría estar de otro modo. En el ejemplo que aquí se ha utilizado, Derek quedaba con poderes intelectuales promedio, lo que sería un estado no comparativamente no malo pero peor que aquél en el que podría haber estado de otro modo. En tal caso, la intuición afirmaba que Derek había sido dañado, pero sostener tal afirmación era difícil para las tesis no-comparativas. La TBEv, en cambio, no encuentra problemas para explicar el daño sufrido por Derek en tal clase de casos. Él ha sido objeto de un evento que lo privó de cierta cantidad de un bien básico, independientemente de si su estado final es bueno o malo. Por tanto, él ha sufrido un daño.

Parece, pues, que la TBEv puede explicar de manera convincente la mayoría de los casos donde se cree que alguien ha sufrido un daño por la acción de otra persona. No obstante, encuentra ciertos problemas, que han sido señalados por J. THOMSON. La primera objeción que realiza, es que HANSER no puede explicar los casos donde los sujetos no pierden ninguna cantidad de un bien, sino que siempre existieron en determinado estado (THOMSON, 2011: 457). La objeción de THOMSON podría ser contestada del siguiente modo. Uno podría afirmar que en tal clase de casos si bien no se priva a nadie de ningún bien, se impide que quien está en determinado estado adquiera cierta cantidad de un «bien básico». Esta respuesta, como se verá luego, dado que — según TBEv no toda vez alguien se vea impedido de recibir un beneficio sufre un daño- no es del todo satisfactoria.

THOMSON realiza una segunda objeción: de acuerdo a la TBEv ser ciego no sería el daño que las personas sufren: el daño sería perder la vista. Esto es implausible ya que de ser así el daño que sufre el sujeto está en el pasado, aunque él siga siendo ciego (2011: 456). La respuesta de HANSER es que es cierto que cuando uno pierde un bien básico es puesto en un estado malo, aunque el daño no derive de tal estado. Sin embargo, la duración de tal estado afecta, dice HANSER, a la gravedad del daño sufrido (2011: 467). Esta respuesta me parece poco convincente. Estoy de acuerdo con THOMSON en que esta respuesta es desconcertante «si la maldad de un evento que consiste en [por ejemplo] la pérdida del uso de las piernas es independiente de [...] la maldad del estado final [...]. ¿Cómo puede HANSER consistentemente decir [...] también que la maldad del evento que consiste en la pérdida del uso de las piernas de alguien es más grave si está en tal estado por un tiempo más largo?» (THOMSON, 2011:457).

Pero supóngase que es posible contestar todas las objeciones a esta clase de tesis. ¿Puede la tesis basada en eventos resolver el problema de la no-identidad? En el caso de Moni y Paola, la TBEv implica lo siguiente: Paola ha perdido la vista a la edad de diez años. Dado que la vista es un bien básico, haberla perdido es sufrir un daño. Moni, ha causado el evento que hizo que Paola pierda la vista. Dado que Moni ha causado el evento que produjo que Paola pierda la vista, ella está en una relación $\mathrm{R}$ con el daño sufrido por Paola. Por tanto, Moni ha dañado a Paola. Parece que el problema de la no-identidad ha sido resuelto. ¿Es esta una buena explicación?

La solución de HANSER a esta clase de casos parece plausible. Estos son los que se han llamado como «casos de malos eventos», aquellos que tienen lugar cuando a las personas les ocurren cosas malas durante sus vidas. En un determinado momento de su vida, ocurre un evento que hace que Paola pierda cierta cantidad de algún bien 
básico. Como los agentes han ocasionado ese evento, ellos están en una relación $\mathrm{R}$ con el daño, y su acción es objetable por ese motivo. Sin embargo, existe otra clase de casos que parece mucho más problemática para aproximaciones como la de HANSER. En los casos de mala condición a las personas no les ocurre nada malo en sus vidas, sino que nacen en esa condición. Imagínese un caso muy similar al de Moni y Paola, sólo que esta vez el niño, en lugar de quedar ciego a la edad de diez años, nace con esa condición. Supóngase, en este caso, que la madre se llama Moni2 y la niña Paola2. ¿Cómo puede la TBEv de HANSER resolver este caso?

El mismo HANSER reconoce que en tal clase de casos es difícil pensar que la niña ciega ha sido dañada por Moni2. Según su tesis, perder un bien básico es sufrir un daño, y Paola2 no ha perdido ningún bien, ella meramente carece del sentido de la vista. «Carecer del sentido de la vista, por contraste no es un sufrir un daño: uno no puede perder lo que nunca se tuvo» (HANSER, 2009: 186). Tal vez HANSER podría acomodar su tesis basada en eventos para cubrir este tipo de casos del siguiente modo. Cuando él explica qué significa sufrir un daño, afirma que la pérdida de un bien básico es sólo una de las formas en las que se puede experimentar el daño. Uno también puede sufrir un daño cuando se le impide recibir algún beneficio. De esta manera uno podría decir que Moni2, al concebir, ha producido un evento que impidió que Paola2 adquiriera cierta cantidad de algún bien básico. Como la concepción, en la forma en que se hizo, impidió que Paola2 tenga el sentido de la vista, la ha dañado. ¿Es esta una buena explicación? Creo que no. La concepción no ha impedido que Paola2 tenga el sentido de la vista. Para que esto sea así debe existir la posibilidad de que ella, de hecho, la tenga. Y, como ya se sabe, tal cosa es imposible en casos atravesados por el problema de la noidentidad. Esta conclusión muestra un problema aún mayor para la TBEv de HANSER. Incluso en los casos de malos eventos, tal como el de Moni y Paola, resulta artificioso afirmar que ha habido un evento que privó a las víctimas de determinado bien básico. Esto es así porque no se puede privar a alguien de lo que nunca pudo haber tenido.

\section{UNA TESIS SUPERADORA, ¿QUÉ ESPERAR DE ELLA?}

Al pretender resolver el problema de la no-identidad a través de alguna noción del daño, han saltado a la vista dos puntos de importancia. El primero, respecto del concepto de daño, es que las personas tienen intuiciones comparativas en algunas clases de casos; en tanto que en otras, sus intuiciones son no-comparativas. Se han explorado dos posiciones que pretendían acomodar estas intuiciones: la tesis combinada y la basada en eventos. Ambas resultaron ser insatisfactorias. El segundo punto, se refiere específicamente al problema de la no-identidad: parece que ninguna de las tesis analizadas, aún si se encontrara un principio que pueda acomodar tales intuiciones encontradas, sería capaz de resolverlo.

Dada la complejidad del asunto y la dificultad de encontrar una explicación plausible, la mejor alternativa es buscar aquella aproximación que más se acerque a las intuiciones «pre-teóricas» que se tienen. En primer lugar, es mayormente aceptado que el estatus de daño que alguien sufre deriva del estado final en el que se está y no tanto de la clase de evento que se sufre. De manera que ese será el punto de partida. 
La aproximación será una basada en estados. En segundo lugar, es bueno tener en cuenta cuáles son los problemas a los que nos hemos enfrentado en este trabajo. Si lo que se pretende es encontrar una aproximación superadora, debería poder resolverlos a todos o, al menos, a la mayor parte. Los problemas en las diferentes tesis analizadas eran los siguientes.

En particular, las tesis comparativas contra-fácticas (TCC y TCC2) no podían resolver (1) el caso de los daños previos, y (2) aquéllos en los que existía una diferencia temporal en el buen efecto y al mal efecto de una acción sobre el mismo aspecto de una persona. Las tesis no-comparativas (TNC), por su parte, encontraban inconvenientes en los casos donde (3) la víctima era colocada en una peor condición de aquélla en la que podría estar de otro modo, pero no en un estado malo. Tampoco podían resolver los casos inversos, es decir cuando (4) el accionar de un agente hace que la víctima mejore su condición, y la coloca en un estado que es mejor de lo que estaría de otro modo, pero ese estado es malo. Una dificultad común a la tesis combinada (TComb) y las no comparativas es (5) mantener al daño como un criterio independiente de cualquier discusión respecto de una distribución de bienes justa como así también (6) explicar cuál es el criterio para conformar el umbral. Respecto de la tesis basada en eventos (TBEv), el mayor problema es que (7) no puede explicar coherentemente cómo se debe graduar la gravedad del daño sin apelar al estado final en el que se encuentran las personas que sufren un daño. Asimismo, un rasgo común a todas las posiciones eran (8) las dificultades para acomodarse al caso de la muerte y (9) la imposibilidad de resolver el problema de la no-identidad. Una aproximación que dé cuenta completamente de las intuiciones que se tienen cuando se afirma que alguien ha dañado a otra persona, debería poder explicar todos estos casos.

\subsection{Reformulación del comparativismo contra-fáctico: TCC3}

Existe una aproximación que puede, creo, ser superadora. Esta tesis es presentada por Judith THOMSON (2011). La autora defiende la idea de que las explicaciones comparativas contra-fácticas, con una leve reformulación, pueden resolver la mayoría de los inconvenientes antes analizados. Su explicación descansa sobre la observación de que el comparativismo contra-fáctico no necesita preguntarse cómo hubiesen sido las cosas para la víctima si no le hubiese pasado lo que pasó (THOMSON, 2011: 448). Sino sólo necesita analizar qué es aquello que la acción del agente impidió que ocurra en la víctima. El ejemplo que ella trae para explicar su punto es el siguiente:

(El villano del ácido) supóngase que un villano A arroja ácido en los ojos de B, causándole ceguera. El ácido comienza a afectar a los ojos de $\mathrm{B}$, pero antes de que se complete el proceso de ceguera de $\mathrm{B}$, una persona allí presente $\mathrm{C}$ interviene aplicando un neutralizador a los ojos de B. Eso hace que B quede en un estado de ceguera débil, llamada «visión borrosa (ThOMSON, 2011: 447).

Según ThOmson cualquiera que piense que B está sufriendo un daño en virtud de tener «visión borrosa» debe abandonar la asunción de que dañar a alguien es causar que alguien sufra un daño (2011: 448), lo que es un problema tanto para las tesis no-comparativas como para las basadas en eventos. THOMSON afirma que cualquier explicación plausible de lo que significa dañar a otro debe implicar que el villano A 
daña a $B$ mientras que $C$ no lo hace, por más que tanto $A$ como $C$ causaron que $B$ tenga «visión borrosa». Conforme a su punto de vista, las tesis comparativas pueden explicar este punto: $\mathrm{A}$ ha causado no sólo que $\mathrm{B}$ tenga visión borrosa, sino que también ha impedido a $\mathrm{B}$ tener buena visión - $\mathrm{y}$ para $\mathrm{B}$ es peor tener una visión borrosa que una buena visión-. Por tanto, A ha dañado a B. En cambio, el obrar de $\mathrm{C}$ aunque también causó que $\mathrm{B}$ tenga visión borrosa, no impidió que $\mathrm{B}$ tenga buena visión. De modo que no lo ha dañado (Thomson, 2011: 448). La tesis de ThOMSON, dado que es también una explicación comparativa contra-fáctica se llamará tesis comparativa contra-fáctica 3 o TCC3:

TCC3: Si A causó que $B$ esté en $\mathrm{E}$, y para algún estado E*: en E, y

i) A impidió que B esté en E* por el mismo medio por el cual A causó que B esté

ii) B está en una peor condición, en algún aspecto, por estar en $E$ de aquélla en la que podría haber estado si hubiese estado en $E^{*}$,

Entonces A daña a B

¿Puede esta tesis solucionar los problemas a los que se enfrentaban las anteriores? Se analizará cada uno de ellos. El primer inconveniente surgía en los casos de (1) daños previos, el cual se ha ejemplificado con el matón y el usurero. La cuestión aquí es poder afirmar convincentemente que el usurero local ha dañado a Joaquín por más que, si él no le hubiese roto las piernas, su víctima hubiese estado en la misma condición porque alguien más (el matón Ramiro) lo hubiera hecho. Este caso es importante porque sirve para observar de un mejor modo la diferencia entre TCC 3 y las tesis comparativas contra-fácticas iniciales ${ }^{34}$.

Como se ha visto, según la Tesis comparativa contra-fáctica clásica (TCC): si el acto de $\mathrm{A}$ hace que $\mathrm{B}$ esté en una peor condición de lo que podría haber estado si $\mathrm{A}$ no hubiese actuado como lo hizo, entonces el acto de A daña a B. En el caso del matón y el usurero, por tanto, siguiendo la TCC se debería concluir que nadie ha dañado a Joaquín. Si el usurero no le hubiese roto las piernas, Ramiro lo hubiese hecho. De modo que el acto del usurero no hace que Joaquín esté en una peor condición de la que de otro modo podría haber estado. En cambio, conforme a la TCC3 no hay obstáculos en sostener que el usurero local ha dañado a Joaquín. El usurero local causó que Joaquín tenga las piernas rotas por: i) el mismo medio que impidió que las tenga sanas, y ii) Joaquín está en un aspecto en una peor condición por tener sus piernas rotas de lo que estaría si las tuviera sanas. De manera tal que el usurero ha dañado a Joaquín por más que este último estaría en la misma condición si él no hubiese actuado como lo hizo. Todo lo que hace falta observar para saber si un sujeto ha dañado o no a otro es la comparación entre el estado de cosas que efectivamente causó y el que impidió que sucediera. Si el primero es peor al segundo, entonces ha habido una acción de dañar.

El segundo problema, que se ha ejemplificado con el caso del Murciélago Negro, surgía en los casos donde (2) un sujeto estaba después de la acción del agente en algún aspecto en una condición peor, pero, pasado un tiempo, en ese aspecto - gracias a aquella acción - estaría aún mejor. La TCC3 en este caso implica lo siguiente: Snate

34 Tim MeIJERs ha objetado que no existen diferencias entre TCC y TCC3. 
causó que Tony esté ciego: i) por el mismo medio que impidió que tenga una visión normal, y ii) mientras Tony está ciego, él está peor que la condición que Snate impidió que esté, es decir, que tenga una visión normal. Dado que la TCC3 descansa en la observación de que el comparativismo contra-fáctico no necesita preguntarse en cómo hubiesen sido las cosas para la víctima si no le hubiese pasado lo que pasó, sino sólo en qué es aquello que la acción del agente impidió que ocurra en la víctima, puede afirmar que Snate ha dañado a Tony por más que, al final de cuentas, esté mejor de lo que podría haber estado de otro modo.

Si las respuestas a (1) y (2) son satisfactorias, la TCC3 se muestra como una alternativa comparativa contra-fáctica superior a las anteriores. Pero ¿es lo suficientemente buena como para resolver otra clase de inconvenientes? Dado que los problemas (3) y (4) lo son sólo para las tesis no-comparativas, no representan un reto para la TCC3. Del mismo modo, dado que es meramente una tesis comparativa no necesita preguntarse por (6) el modo en que debe construirse el umbral o qué clase de estados deben catalogarse como malos. Por tanto no necesita hacer consideraciones referentes al problema de la justicia (5). Respecto de la tesis basada en eventos (TBEv) su mayor problema era (7) no tener un criterio coherente para graduar la gravedad del daño. Conforme a la TBEv el estado final en el que están las personas no cuenta para afirmar si han sido dañadas o no; aunque a la vez sí cuenta para medir su gravedad. La TCC3 no tiene este problema, puede graduar la gravedad del daño en proporción a cuán mala sea la condición en la que la víctima ha sido colocada en relación con aquella otra condición en la que le impidió estar.

Si las afirmaciones anteriores son convincentes se ha encontrado una tesis de lo que significa dañar a otro que es superior no sólo a la original TCC, sino a todas las otras analizadas. Sin embargo, ¿puede ella resolver el problema (8) de la muerte y (9) el de la no-identidad? Respecto del caso de la muerte (8) tal y cómo se ha construido la TCC3 es difícil explicar por qué estar muerto es malo. Podría afirmarse que en ese específico caso es cierto que cuando se mata a una persona, uno no causa que la vida de la víctima este en una mala condición (o sea mala en algún sentido), sino sólo que se acabe (THOMSON, 2011: 455). Lo que aunque parece ser algo malo también es un asunto diferente. No creo que esta afirmación sea completamente satisfactoria para explicar el daño sufrido. Pero, dado que tampoco alguna de las otras tesis puede explicar este caso, eso no altera la consecución de los objetivos de este trabajo. Esos objetivos eran encontrar una explicación de lo que implica dañar a otro superior a las otras y de tratar, a través de ella, resolver el problema de la no identidad.

\subsubsection{TCC3 y el problema de la no identidad}

Sin embargo, ¿puede la TCC3 resolver el problema de la no-identidad? Para responder esta pregunta se debe distinguir entre los casos que directamente involucran al problema de la no-identidad y a aquellos que lo hacen indirectamente. En la primera clase de casos una acción genera un curso causal que produce una consecuencia mala en un ser a la vez que su existencia. En cambio en la segunda clase de casos una elección tiene consecuencias que inician un complejo de cadenas de eventos que eventual- 
mente tienen un igual efecto decisivo sobre quienes serán las personas particulares que existirán después de haber sido hecha la elección. En esta segunda clase de caso la contribución de cada acción en la producción del resultado es menor. Se comenzará con los casos que indirectamente involucran al problema. El ejemplo típico de esta clase de casos es el siguiente:

(La Política Catastrófica) ${ }^{35}$ : Una comunidad tiene que elegir entre dos políticas energéticas. Como resultado de la elección de cada política, las personas que vivirán en cada escenario posible serán diferentes. Las dos políticas serían completamente seguras por al menos tres siglos, pero se sabe que una de ellas causará una catástrofe en un futuro más lejano. Una política, la política catastrófica supone el enterramiento de desechos nucleares en áreas en que no hay riesgos de terremoto, bajo la modalidad de almacenamiento temporal prolongado. Esta forma de almacenamiento permite guardar los desechos de manera segura entre 100 y 300 años. Pero como estos desechos seguirán radiactivos durante miles de años, es previsible que pasado ese periodo se produzca un escape, lo que ocasionará una catástrofe ecológica. Si se elige esta Política Catastrófica, el nivel de vida será algo más alto en el próximo siglo. Por eso la comunidad la elige. Como resultado, hay una catástrofe muchos siglos después. Se libera una radiación que afecta a personas que nacen después del escape, a las que les hace contraer una enfermedad incurable - $v$. gr. leucemia - a la edad de 40 años. Supóngase que una de las personas afectadas, llamémosle Hulk, no habría vivido si se hubiese elegido la política segura, la que se desarrolla bajo la forma del almacenamiento geológico profundo.

¿Cómo opera la TCC3 en tal situación? Según THOMSON en esta clase de casos (los que indirectamente implican el problema de la no-identidad) se disparan al menos dos procesos causales diferentes. Uno que va hacia el nacimiento de Hulk (y otros niños); el otro hacia la adquisición de leucemia por parte de Hulk como consecuencia de la catástrofe producida siglos después (2011: 453). Esto es así porque tanto uno como otro resultado se produce como consecuencia de la elección de la política catastrófica en conjunción con otros actos que o no han sido influenciados por tal elección o lo han sido en diferente grado. Si esto es aceptable, elegir la política catastrófica, en primer lugar, ha provocado una cadena causal cuyo resultado fue que Hulk exista por el mismo medio que impidió su inexistencia. En segundo lugar, dado que se está hablando de dos cadenas causales diferentes, es posible afirmar que respecto de la segunda, elegir la política catastrófica produjo que Hulk tenga leucemia por el mismo medio que impidió llevar una vida saludable. Si esto es así, puede afirmarse que él ha sido dañado. La acción disparó un proceso causal que impidió que Hulk esté en una posición que es mejor que aquella otra que causó.

Puede objetarse que esta distinción entre diferentes cadenas causales es más artificiosa que real, es decir que la solución propuesta sería algo así como una trampa lingüística. Sin embargo, de acuerdo con el método estándar para evaluar contra-fácticos, un contra-fáctico dado es verdadero si y sólo si su consecuencia es verdadera en el mundo posible «más cercano» —el mundo más similar al mundo real— (HANSER, 2009: 197). El mundo posible más cercano a aquel en el que Hulk contrae una enfermedad, es aquel en el que la catástrofe no ocurre y Hulk de todos modos nace ${ }^{36}$. Y no

35 He modificado el ejemplo de la política arriesgada de PARFIT (1984: 371).

36 Alan CARTER proporciona un argumento similar combinándolo con una tesis inversa de la efectos acumulativos de las acciones (CARTER, 2001: 441-442). 
aquel donde además de lo primero, Hulk no nace. Esto es así porque cada resultado se produce como consecuencia de la combinación de la elección de la política catastrófica con hechos diferentes.

Pero, ¿puede la TCC3 explicar por qué Moni ha dañado a Paola? Desafortunadamente, creo que la respuesta es negativa. Este caso es uno en el que directamente se ve implicado el problema de la no-identidad. En estos casos, una acción genera sólo un curso causal que produce dos (o más consecuencias): una buena, que nazca algún ser; y una mala, que tenga alguna discapacidad. Aquí, a diferencia del caso anterior, el mundo posible más cercano al real donde Paola no tiene esa discapacidad es aquel en el que ella no nace. La prueba de esto es que el nacimiento de Paola y su ceguera tienen exactamente las mismas causas. A diferencia del caso de la política catastrófica, donde si bien su elección es causa tanto del nacimiento de Hulk como de que contraiga una enfermedad, cada uno de los resultados se produce como consecuencia de la conjunción de aquella elección y diferentes causas.

\section{CONCLUSIÓN}

Este trabajo comenzó puntualizando que, en general, en los casos atravesados por el problema de la no-identidad, se tiene la intuición que las acciones presentes pueden dañar a las personas futuras. Se intentó mostrar que las explicaciones del daño comúnmente aceptadas no pueden apoyar esa clase de juicios. Por tal razón, se buscó una tesis del daño superadora que pueda resolver también los casos atravesados por el problema de la no-identidad. El inconveniente se esquematizó del siguiente modo:

P1: Si el acto de A hace que B está en una peor condición de la que podría haber estado si A no hubiese actuado como lo hizo, entonces el acto de A daña a B.

P2: El acto de Moni de concebir ahora en lugar de tomar la píldora por dos meses antes de concebir no hace que Paola esté peor de lo que podría estar si Moni no hubiese actuado como lo hizo.

C: Por consiguiente, el acto de Moni no daña a Paola.

En este trabajo se buscó una respuesta que evite la conclusión en términos del daño a los seres futuros, es decir que niegue a P1 y que cumpla, siguiendo a BoONIN, con los requisitos de: (1) independencia, (2) robustez y (3) modestia. El requisito de la independencia (1), implicaba que la razón para rechazar la P1 debía ser independiente del hecho de que rechazar la $\mathrm{P} 1$ es lo que nos permite evitar la conclusión. El requisito de la robustez (2) implicaba que la razón para rechazar P1 debía ser una buena razón para rechazar la conclusión por sí misma. Por último, el requisito de la modestia (3) implicaba que la razón para rechazar la conclusión no debía generar situaciones o problemas en los que se tengan incluso más razones para evitar, que las que se tenga para evitar la CE.

El objetivo propuesto no ha podido ser cumplido. No se ha encontrado una explicación del daño que sea superior a las otras y a su vez pueda resolver completamente el problema de la no-identidad. Sin embargo, a través del análisis realizado se ha podido defender que una reformulación de las tesis clásicas comparativas contra-fácticas puede resolver casi todos los inconvenientes que presentan las demás explicaciones del 
daño. La tesis defendida, TCC3 dado que puede resolver gran cantidad de inconvenientes en casos no atravesados por el problema de la no-identidad que son problemáticos para la tesis implicada en P1, demostró — hasta ahora - ser superadora. Por tal razón se defendió que la TCC3 es capaz de rechazar a la implicada en P1, cumpliendo de ese modo, con el requisito de la independencia.

Asimismo, se defendió que no hay mayores razones para evitar las conclusiones a las que TCC3 arriba, tanto en casos atravesados por el problema de la no-identidad como en aquellos donde no tiene presencia, que las que existen para evitar la conclusión de nuestro silogismo. Prueba de esto es que la TCC3 da la respuesta correcta en al menos cierta clase de situaciones atravesadas por el problema de la no-identidad; y donde no da la respuesta correcta, afirma lo mismo que la tesis comparativa clásica. Lo mismo ocurre con los casos no atravesados por el problema de la no-identidad. Como esto es así, el requisito de la modestia ha sido cumplido.

Sin embargo, la TCC3 no es lo suficientemente robusta como para rechazar la conclusión por sí misma. Prueba de ello es que no se ha podido explicar por qué en los casos que directamente implican el problema de la no identidad, como el de Moni y Paola, el agente que actúa daña a la persona sobre la cual las consecuencias acaecen. Precisamente, casos como el de Moni y Paola, se presentan como los más problemáti$\cos ^{37}$. Sin embargo, como la TCC3 no deja sin resolver casos que las otras tesis sí, debe preferirse y ser adoptada. $\mathrm{Al}$ menos hasta que se proponga una salida superadora que pueda resolver el problema de la no-identidad.

\section{BIBLIOGRAFÍA}

Adams, R. M., 1979: «Existence, Self Interest, and the problem of evil», Nô̂s, vol. 3, núm. 1, 53-65.

Boonin, D., 2014: The Non-Identity Problem and the Ethic of Future People, Oxford University Press.

Brock, D., 1995: «The Non-Identity Problem and Genetics Harms», Bioethics, vol. 9, núms. 3-4, 269-275.

CARTER, A., 2001: «Can we Harm Future people?», Environmental Values, vol. 10, núm. 4 (noviembre 2001), 429-454.

FEINBERG, J., 1984: Harm to others: the moral limits of criminal law, vol. I, New York: Oxford University Press.

- 1988: Harmless Wrongdoing: the moral limits of criminal law, vol. IV, New York: Oxford University Press.

- 1994: «Wrongful Life and the Counterfactual Element in Harming», en id., Freedom and Fulfillment: philosophical essays, New Jersey: Princeton University Press, 3-36.

Gosseries, A., 2008: «On Future Generations’ Future Rights», The Journal of Political Philosophy, vol. 16, núm. 4, 2008, 446-474.

37 Aunque estos casos sean teóricamente más problemáticos, no parecen ser los que tengan mayor importancia práctica. Parece que la mayoría de los casos que pueden presentarse donde el problema de la no-identidad está presente son aquellos que indirectamente lo implican. Esto es así porque los efectos de cada acción se dispersan cada vez más y más a lo largo del tiempo, de manera que pueden contribuir en diferentes cadenas causales a la producción de diferentes resultados. Romina FRONTALINI REKERS me ha hecho notar este dato. 
Hanser, M., 1990: «Harming Future People», Philosophy and Public Affairs, vol. 19, núm. 1, 47-70.

- 2008: «The Metaphysics of Harm», Philosophy and Phenomenological Research, vol. LXXVII, núm. 2, 421-450.

— 2009: «Harming and procreating», en M. ROBERTS y D. WASSERMAN (eds.), Harming Future Peoples: Ethics, Genetics and the Non Identity Problem, New York: Springer, 179-199.

- 2011: «Still more on The Metaphysics of Harm», Philosopby and Phenomenological Research, vol. LXXVII, núm. 2, 459-469.

Harman, E., 2004: «Can we harm and benefit in creating?», Philosophical Perspectives, 18, Ethics, 2004, 89-113.

— 2009: «Harming as Causing Harm», en M. RoBerTS y D. WASSERMAn (eds.), Harming Future Peoples: Ethics, Genetics and the Non Identity Problem, New York: Springer, 137-151.

Holtung, N., 2002: «The Harm Principle», Ethical Theory and Moral Practice, vol. 5, núm. 4 (diciembre 2002), 357-389.

Hurley, P., y Weinberg, R., 2014: «Whose Problem is Non-Identity?», Journal of Moral Philosophy, abril 2014.

McMahan, J., 1981: «Problems of Population Theory», Ethics, 92, núm. 1 (octubre 1981), 96127.

— 1998: «Wrongful Life: Paradoxes in the Morality of Causing People to Exist», en J. CoLEMAN y C. MORRIS (eds.), Rational Commitment and Social Justice: Essays for Gregory Kavka, Cambridge University Press, 208-247.

- 2012: «Causing People to Exist and Saving People’s Lives», Ethics, Springer, 13 de septiembre de 2012.

Meyer, L., 2003: «Past and Future: The Case for a Threshold Notion of Harm», en L. Meyer, S. Paulson y T. Pogge, Rights, Culture, and the Law: Themes from the Legal and Political Philosophy of Joseph Raz, Oxford University Press, 143-159.

- 2015: «Intergenerational Justice», The Stanford Encyclopedia of Philosophy (Winter 2015 Edition), en E. N. ZALTA (ed.), bttp://plato.stanford.edu/archives/win2014/entries/justiceintergenerational/.

Meyer, L., y Roser, D., 2009: «Enough for the Future», en A. Gosseries y L. Meyer, Intergenerational Justice, Oxford University Press, 219-248.

MiLl, J. S., 1992: Sobre la Libertad, Madrid: Alianza (trad. P. DE AzCÁRATE).

NinO, C. S., 1980: Los límites a la responsabilidad penal: una teoría liberal del delito, Buenos Aires: Astrea.

- 2013: Ocho lecciones de ética y derecho para pensar la democracia, Buenos Aires: Siglo XXI.

PARFIT, D., 1976: «On doing the Best for Our Children», en M. BAYLES (ed.), Ethics and Population, Cambridge: M. A. Shenkmann, 100-115.

- 1984: Reasons and Persons, Oxford: Clarendon Press.

— 1986: «Comments», Ethics, vol. 96, núm. 4 (julio de 1986), 832-872.

— 1988: «What we together do», Unpublished, 29 de marzo de 1988, 1-33.

Petersen, T. S., 2014: «Being Worse Off: But in Comparison with What? On the Baseline Problem of Harm and Harm Principle», Res Pública, 27 de febrero de 2014, Springer, 1-16.

RAZ, J., 1986: The Morality of Freedom, Clarendon: Oxford University Press.

- 1987: «Autonomy, Toleration and the Harm Principle», en R. GAVISON (ed.), Issues in Contemporary Legal Philosophy, Oxford: Clarendon Press, 315-333.

Reiman, J., 2007: «Being Fair to the Future People: The Non-Identity Problem in the Original Position», Philosophy \& Public Affairs, 35, núm. 1. 
Rivera López, E., 2011: «Responsabilidad Procreativa y el Problema de la No Identidad», en id., Problemas de vida o muerte: Diez ensayos de bioética, Barcelona: Marcial Pons, 93-123.

RoBerTs, M., 2007: «The non-identity Fallacy: Harm, Probability and Another Look at Parfit's Depletion Example», Utilitas, vol. 19, núm. 3, septiembre de 2007, Cambridge University Press, 267-311.

- 2009: «The non-identity problem and the two Envelope problem: When is One act Better for a Person than Another?», en M. RoberTS y D. WASSERman (eds.), Harming Future Peoples: Ethics, Genetics and the Non Identity Problem, New York: Springer, 201-228.

SCHWARTZ, T., 1979: «Welfare Judgments and Future Generations», Theory and Decision, 11 (1979) $181-194$.

SHIFFRIN, S. V., 1999: «Wrongful Life, procreative responsibility and the significance of harm», Legal Theory, vol. 5 (1999), Cambridge University Press, 117-148.

- 2012: «Harm and Its Moral Significance», Legal Theory, vol. 18, septiembre (2012), Cambridge University Press, 357-398.

SMAJDOR, A., 2014: «How Useful is the concept of the "Harm Threshold in reproductive ethics and law"?», en Theor Med Bioeth, Springer, 9 de agosto de 2014, 1-16.

Thomson, J. J., 2011: «More on the Metaphysics of Harm», Philosophy and Phenomenical Research, vol. LXXXII, núm. 2, 436-458.

WoOdWARD, J., 1986: «The Non-Identity Problem», Ethics, vol. 96, núm. 4 (julio de 1986), 804-831.

— 1987: «Reply to Parfit», Ethics, vol. 97, núm. 4 (julio de 1987), 800-816. 